\title{
Lost in Translation: Limitations of a Universal Approach in Genetic Counseling
}

\author{
Hamid Pour-Jafari • Bahareh Pourjafari
}

Received: 6 May 2009 / Accepted: 26 August 2009/Published online: 3 October 2009

(C) National Society of Genetic Counselors, Inc. 2009

Keywords Genetic counseling · Diversity · Ethics .

Universal approach

In countries with a well-developed genetic counseling profession, genetic counselors receive specialized graduate training that includes the study of genetic diseases and how those diseases run in families, and supervised genetic counseling practice. In addition they usually work as part of a health care team in conjunction with other trained specialists to help families make informed decisions about their genetic risk. They also work as patient advocates, helping individuals receive additional support and services for their health care needs. In contrast, in some developing countries physicians, human geneticists, and nurses who do not have specialized training in all of these domains, provide genetic counseling services. And in some cases, they have no academic training courses specific to genetic counseling.

Genetic counseling is a complex activity requiring counselors to not only have extensive knowledge of human genetics and well-honed counseling skills, but also to be able to apply their knowledge and skills in ethically and

H. Pour-Jafari $(\bowtie) \cdot$ B. Pourjafari

Research Center for Molecular Medicine,

Hamadan University of Medical Sciences,

PO Box 518, Hamadan, Iran

e-mail: h_pourjafari@yahoo.com

e-mail: pourjafari@umsha.ac.ir

H. Pour-Jafari

Molecular Medicine and Genetic Department,

School of Medicine, Hamadan University of Medical Sciences,

Hamadan, Iran culturally sensitive ways. For instance, genetic counselors should tailor their interventions to each client's situation, discussing the client's family history and test results in detail, explaining when and how genetic testing may not be appropriate for their situation, and informing them of other options to reduce their risk or lifestyle changes that may help their situation. Sometimes certain options clash with client's cultural and/or religious beliefs (e.g., having ovaries removed in women at risk for ovarian cancer, or raising termination of a pregnancy as an option), and counselors should be able to anticipate these conflicts.

Another facet of the complexity of genetic counseling is that it is a two-way process; the only way counseling can be effective is if clients engage in ways that allow genetic counselors to understand them, that is, disclosing all relevant information to the best of their knowledge, and being open to exploring options. In an ideal genetic counseling process, the client trusts the counselor (and other medical team members who often participate in designing an action plan for continued medical management), and thus feels comfortable providing information and participating in decision-making. To promote this ideal goal, genetic counselors should be familiar with their clients' socioeconomic situations, cultures, religions, values, and so on.

There is a substantial and growing body of genetic counseling literature. A majority of these books and scientific papers are authored by western scientists, and usually they are most helpful for practitioners and clients in their own countries. Little explicit attention is given to the fact that those materials, in almost all cases, are translated verbatim into other languages and considered as "standards" for practice in developing countries, despite the often deep differences among cultures. An obvious risk of directly 
generalizing this literature to genetic counseling practice in non-western countries is that the counseling will be ineffective. Furthermore, generalization of certain literature to other western countries may also be problematic with respect to its accuracy and utility (e.g., the United States and Canada have different health care systems). Moreover, even generalization of findings within the region in which a study was conducted is limited in countries that have multicultural populations (e.g., Australia, U.S., Canada).

Thus, we challenge the idea that a "universal protocol" for genetic counseling can and should be developed from current books and scientific articles. Instead, we urge theorists, researchers, and educators to explicitly articulate those aspects of genetic counseling that are relatively universal, and those aspects which are culture-specific. We would like to see more coordinated international efforts that bring together scientists, practitioners, and other interested parties for discussions and eventual resolution of challenges posed by the provision of genetic services.
Topics of relevance for such international dialogues include:

1. Building and strengthening an international organization for genetic counseling.

2. Standardization of genetic counseling education through the world.

3. Developing culture-based protocols for genetic counseling (especially in some sensitive subjects, such as termination of a pregnancy, prenatal diagnosis, male and female sterility, pre-implantation sex selection, IVF, embryo donation, sperm donation).

4. Conceptualizing culture-based ethics in genetic counseling: necessities, possibilities and difficulties.

We further suggest the World Health Organization (WHO) provide leadership in these international efforts. The WHO, a specialized agency of the United Nations, serves as a coordinating authority on international public health (Wikipedia, 2009). 